\title{
Power-Law Congestion Costs: Minimal Revenue (MR) Pricing and the Braess Paradox
}

\author{
Claude M. Penchina* \\ Department of Physics, Hasbrouck Laboratory, University of Massachusetts at Amherst, Amherst Massachusetts 01003 \\ USA
}

Department of Physics, King's College, Strand, London, WC2R-2LS, UK

ECE Department of UCSD, La Jolla, CA 92093, USA

\begin{abstract}
We describe a simpler proof for Calvert and Keady's (C-K) theorem showing the non-occurrence of the Braess Paradox in networks with power-law congestion costs. We extend the C-K theorem to the case of elastic demand. We then use the methods of these proofs to develop several new theorems about the optimality of flows and the piecewise stability of Minimal Revenue (MR)Tolls in transportation networks with power-law congestion costs, with and without fixed costs. The stability of MR tolls is an important attractive feature. For administrators it makes the tolls cheaper to collect. For users it makes the tolls more predictable.
\end{abstract}

Keywords: Braess's paradox, congestion costs, network theorems, power law non-linearities wheatstone bridge.

\section{INTRODUCTION}

Link cost functions used in transport network modeling usually include fixed costs plus variable congestion costs. The congestion costs are often modeled as powers of the flow; e.g., 1st power (linear) and 4th power are both popular choices. Calvert and Keady [1], hereinafter referred to as C$\mathrm{K}$, proved a theorem that the Braess Paradox [2] can not occur in networks in which all links have no fixed costs and all congestion costs are a single power law. We provide here a simpler proof of the C-K Theorem. Since our proof is based on rather general optimization ideas in traffic networks, it provides additional insight into the basic reasons behind the theorem. Although our proof is shown using traffic assignment methods in a transport network, the results are quite general for any networks satisfying the equivalent of the Kirchhoff Voltage Rule (essentially equivalent to Wardrop's First Principle) and the Kirchhoff Current Rule (conservation of flow) for electric circuits [3,4]. Furthermore, the new method of proof leads to additional new theorems concerning the stability of Minimal-Revenue (MR)Tolls under changing network flow conditions.

Braess [2] described a traffic flow network in which an extra arc (link, road) that was added in order to relieve congestion, counter-intuitively increased the travel time for all users of the network. C-K restated the paradox for physical networks. "BRAESS'S PARADOX. The power consumed in a nonlinear network can increase if an arc's conductivity is increased with consumptions [corresponding to demands in traffic networks] held constant." In electrical networks, a more intuitive way of stating it might be: BRAESS'S PARADOX. Decreasing the resistance in a branch of the

*Address correspondence to this author at the Department of Physics, Hasbrouck Laboratory, University of Massachusetts at Amherst, Amherst Massachusetts 01003, USA; E-mail: cmpenchina@gmail.com circuit can decrease the current at fixed input voltage, or increase the voltage at fixed input currents. C-K were particularly concerned with physical water-supply pipe networks. We are mainly concerned with traffic networks. The results apply equally to any network with "a potential function defined over the nodes and a flow defined over the arcs" [1]. Examples of such networks and their corresponding terminologies are in Table 1. Note that in traffic networks, a node's potential equals the "length" (total generalized cost, $\mathrm{GC}$, including tolls) of the min-GC path from the origin node to it (see Dial [5] p. 191).

\section{BRIEF OUTLINE OF THIS PAPER}

SECTION 2 reviews the $\mathrm{C}-\mathrm{K}$ theorem on the nonexistence of the Braess Paradox for power-law congestion costs.

SECTION 3 provides a simpler proof of the C-K theorem, the method of proof being useful for deriving new theorems.

SECTION 4 shows that MR tolls are zero for inelastic demand with power-law costs.

SECTION 5 shows that costs including fixed costs plus power-law congestion costs are sufficient (Theorem 4), but not necessary (Theorem 3) to make Minimal Revenue tolls piecewise stable for inelastic demand. This section is presented in two major parts. Part 5A uses simple examples to prove Theorem 3, and suggest the truth of Theorem 4. Part $5 \mathrm{~B}$ is a general proof of Theorem 4.

SECTION 6 summarizes the results.

\section{CALVERT-KEADY (C-K) THEOREM 1 FOR TYPE (H) NETWORKS.}

In order to compare more easily with $\mathrm{C}-\mathrm{K}$, we introduce some of their notation here. C-K "define a network to be of 
Table 1. Equivalent Terms for Various Networks

\begin{tabular}{|l|c|c|c|c|}
\hline \multicolumn{1}{|c|}{ Network } & Traffic & Water & Electrical & Thermal \\
\hline \hline Node, Vertex & Intersection & Joint & Junction, node & Junction \\
\hline Arc, Edge, Link & Road, Link & Pipe & Branch & Temperature $=\mathrm{T}$ \\
\hline Potential & Time or GC & Head $=\mathrm{p}$ & Voltage $=\mathrm{V}$ & Heat Sink, Reservoir \\
\hline Reference Potential (zero) & Origin & Sea Level & Ground, Earth & Heat-Flow $=\mathrm{Q}$ \\
\hline Flow & Traffic Flow & Water Flow & Current $=\mathrm{I}$ & Heat Input \\
\hline Consumptions & Demands & Source, Supply & Input Currents & $\Delta \mathrm{T} / \mathrm{Q}$ \\
\hline Resistance & Time or GC per unit flow & P / Water Flow & $\Delta \mathrm{V} / \mathrm{I}$ & $\mathrm{Q} \times \Delta \mathrm{T}$ \\
\hline Power= Flow x $\Delta$ Potential & Vehicle Hours, System Cost & Flow $x \mathrm{p}$ & Power $=\mathrm{I} \times \Delta \mathrm{V}$ & \multicolumn{2}{|c|}{} \\
\hline
\end{tabular}

In traffic networks GC represents Generalized Cost $=$ Time plus the time-equivalent of other costs

Note: A node's potential equals the "length" (total generalized cost, GC, including tolls) of the min-GC path from the origin node to it (see Dial [5] p. 191).

type $(\mathrm{H})$ if, for each arc $\mathrm{A}$, the flow is a numerical factor $\mathrm{k}_{\mathrm{a}}$ times the head difference to a power $\mathrm{s}>0$ independent of a" [1] . Equivalently,

DEFINITION. Type (H) Network $\Delta \mathrm{p}_{\mathbf{a}}=\mathrm{g}_{\mathbf{a}}\left(\mathrm{x}_{\mathbf{a}}\right)^{\mathrm{n}}$

where $\mathrm{x}_{\mathbf{a}}$ is the flow through $\operatorname{arc} \mathrm{A}, \Delta \mathrm{p}_{\mathbf{a}}$ is the potential difference across $\mathrm{A}, \mathrm{n}=1 / \mathrm{s}>0$ and $\mathrm{g}_{\mathbf{a}}=\left(1 / \mathrm{k}_{\mathbf{a}}\right) \mathrm{n}$.

$\mathrm{C}-\mathrm{K}$ then prove "THEOREM 1" which we paraphrase as saying: the Braess Paradox does not occur for networks with single-power-law link costs that satisfy the Kirchhoff rules.

Since type H networks have no fixed costs, they may not be good examples of real traffic networks, except in cases of very heavy congestion for which the fixed costs may be negligible compared to the congestion costs. Still, these networks form a good starting point for a later discussion of more realistic networks that have both fixed costs and congestion costs.

Note that electric, water, and thermal network theories traditionally allow for negative flows, whereas traditional traffic network theories usually constrain the flow to be nonnegative (one-way road lanes). Real traffic flows can occasionally be negative, but often at high cost (e.g. injury, fines). Since the usual assumptions of traditional traffic network theories are in any case fairly crude approximations to real life traffic, it is justifiable to use Kirchhoff's rules in traffic theories as is done for the other networks, but with usually high reverse "resistance".

This allows for use of mathematical techniques, developed over many years for other networks, to be used for traffic networks too.

\section{NEW PROOF OF THE CALVERT-KEADY (C-K) THEOREM I -- NO BRAESS PARADOX FOR POWER-LAW COSTS}

A very brief informal proof states that for power law costs, the tolls used to induce System Optimal (SO) flows would multiply each link cost by the same factor, thereby not changing the flow pattern -- so the User Optimal (UO) flows are already SO, and thus not paradoxical.

A more formal, exposition of this proof follows. This formal version, still much simpler than that of $\mathrm{C}-\mathrm{K}$, is useful because the methods to develop the proof are used here again to prove additional theorems.

It was noted by a reviewer, that: Theorem 1 was also presented earlier in the economics literature by Barro and Romer [6]; also, Cowen and Glazer [7] point out in a comment on the Barro-Romer paper, that the result is also well known in the theory of clubs (3a) We consider a traffic network of type $(\mathrm{H})$. The potential difference across link $\mathrm{j}$ is its cost function $\mathrm{c}_{\mathrm{j}}$, which depends on the flow $\mathrm{x}_{\mathrm{j}}$ through it:

$c_{j}=g_{j}\left(x_{j}\right)^{n}$

It is well known in traffic networks $[5,8]$ that marginal cost (MC) tolls induce User Optimizing (UO) drivers to voluntarily chose System Optimized (SO) routes. Here SO means that the total system cost (power, vehicle-hours) is minimized for inelastic demand. Note that tolls are not considered system costs, since they are just a redistribution of wealth within the system.

System Cost $=\sum_{j} c_{j} x_{j}$

The Marginal Cost (MC) Toll, Tmc, on Link $\mathrm{j}$ is

$\mathrm{Tmc}_{\mathrm{j}}=\mathrm{x}_{\mathrm{j}} \partial \mathrm{c}_{\mathrm{j}} / \partial \mathrm{x}_{\mathrm{j}}$

so from $\mathrm{Eq}(3.1)$

$\mathrm{Tmc}_{\mathrm{j}}=\mathrm{ng}_{\mathrm{j}}\left(\mathrm{x}_{\mathrm{j}}\right)^{\mathrm{n}}$

The Difference in Potential $\Delta \mathrm{p}_{\mathrm{j}}$ across link $\mathrm{j}$ is the Generalized Cost (GC) of link $j$

$\Delta \mathrm{p}_{\mathrm{j}}=\mathrm{GC}_{\mathrm{j}}=\mathrm{c}_{\mathrm{j}}+\mathrm{Tmc}_{\mathrm{j}}=(\mathrm{n}+1) \mathrm{g}_{\mathrm{j}}\left(\mathrm{x}_{\mathrm{j}}\right)^{\mathrm{n}}=(\mathrm{n}+1) \mathrm{c}_{\mathrm{j}}=\mathrm{N} \mathrm{c_{ \textrm {j } }}$

(3b) The generalized cost GC with MC tolls is simply a numerical factor $\mathrm{N}=(\mathrm{n}+1)$ times the original cost without tolls.

(3c) From (3b), by symmetry, the relative distribution of flows remains unchanged by the MC tolls. If this is not immediately, evident, consider any two paths between any two nodes. If the flow in each path changes with time by the same factor $m$, the costs for each path change by a factor $\mathrm{Nm}^{\mathrm{n}}$. Thus, costs that were equal on the two paths in user equilibrium without tolls, remain equal with tolls. The relative flows on the two links are unchanged, the individual flows each changing by the same factor $\mathrm{m}$. 
(3d) If the demands are inelastic, from (3c) the absolute flows remain unchanged by the $\mathrm{MC}$ tolls.

(3e) It is well known that the flows with MC tolls are SO.

(3f) Thus, from (3d) and (3e) the original flows without tolls were also SO.

Thus we have proved

THEOREM 1A. INELASTIC DEMAND - OPTIMALITY OF TYPE H NETWORK FLOWS - For networks with singlepower-law link costs that satisfy the Kirchhoff rules (Type $H)$, and have inelastic demands, the UO flows in the network are system-optimal (SO).

Stated in other words, the UO flow pattern is the same as the SO flow pattern when the link cost contains negligible free flow travel time (and/or other fixed costs), and the congestion costs are all the same power law functions of the flow.

$(3 \mathrm{~g})$ Now, consider a used link "B" in the network (flow $\mathrm{x}_{\mathrm{b}}>0$ ) that is SUSPECTED OF BEING PARADOXICAL, in the (reverse) Braess sense that removing it would decrease each individual user's cost. Removing the link is equivalent to forcing that link flow $\mathrm{x}_{\mathrm{b}}$ to be zero. However, by (3f), changing any flow (either increasing or reducing it) would destroy the SO flow pattern. Hence, the suspected link is NOT PARADOXICAL. This argument holds for each link in turn throughout the network -- so there are no paradoxical links and thus no Braess Paradox for this power-law network, and we have proved theorem 1B. (See also ref. [9]).

THEOREM 1B. INELASTIC DEMAND - NO BRAESS PARADOX IN TYPE H NETWORKS- The Braess Paradox does not occur for networks with single-power-law link costs that satisfy the Kirchhoff rules (Type H), for inelastic demands.

(3h) Now, consider the case of ELASTIC DEMAND. Using a graphical analysis, it was shown by Penchina [10] that if the Braess Paradox occurs for inelastic demand, it also occurs for elastic demand.

For convenience of the reader, Fig. (2) for a "minimal critical network" from the 1997 paper by Penchina [10] is reproduced here as Fig. (2) for an example of cost- $v s$-flow curves which can cause the Braess Paradox. We have also added a downward sloping dash-dot line as an example of elastic demand, and a vertical dotted line as an example of inelastic demand. The occurrence of the paradox requires the Cost $v s$ Flow curve with the added link (solid line with arrows on it) to be above the curve without the added link (upward sloping dashed line) for some values of flow. The paradoxical region is that region (a triangle in the example of Fig. (2)) where the solid line Cost vs Flow with added link is above the dashed Cost vs Flow without the added link. Any demand curve that goes thru the paradoxical region will lead to the occurrence of the paradox. The non-occurrence of the paradox with any inelastic demand (Theorem 1A) implies that the cost- $v s$-flow curve with the added link is never above the one without the added link. Thus, there is no paradoxical region, so no elastic demand curve can cross through a paradoxical region; i.e. the paradox can not occur, and we have proved Theorem $1 \mathrm{C}$.

$$
\text { MinRevToll fig } 1 \quad \text { Claude M Penchina (c)2000 }
$$

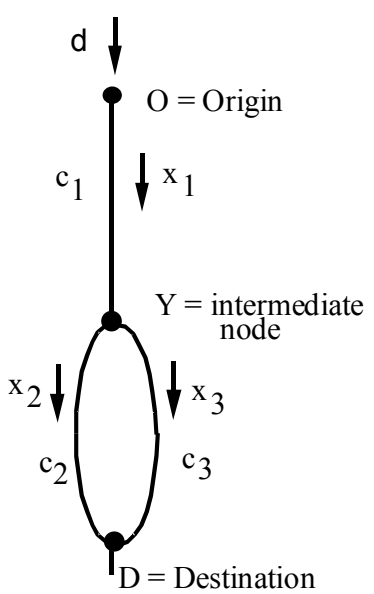

$$
\begin{array}{ll} 
& \begin{array}{l}
\text { Symbols } \\
\mathrm{d} \quad
\end{array} \\
\text { demand } \\
\mathrm{h}_{\mathrm{j}} \quad \begin{array}{l}
\text { Fixed Cost } \mathrm{h} \\
\text { (time plus toll) on link } \mathrm{j}
\end{array} \\
\mathrm{g}_{\mathrm{j}} \quad \begin{array}{l}
\text { Congestion cost } \mathrm{g}, \\
\text { per unit flow } \mathrm{x} \text {, on link } \mathrm{j}
\end{array} \\
\mathrm{x}_{\mathrm{j}} \quad \begin{array}{l}
\text { Flow (link load) } \mathrm{x} \\
\text { on link } \mathrm{j} .
\end{array} \\
\mathrm{c}_{\mathrm{j}} \quad \begin{array}{l}
\text { Total user cost } \mathrm{c} \text { on link } \mathrm{j} \\
\mathrm{c}_{\mathrm{j}}=\mathrm{h}_{\mathrm{j}}+\mathrm{g}_{\mathrm{j}} \mathrm{x}_{\mathrm{j}}
\end{array}
\end{array}
$$

Fig. (1). A simple 3-link, 2-path, 1-origin, 1-destination network used to illustrate User Optimal (UO), and System Optimal (SO) flows with Marginal Cost (MC) Tolls and Minimum Revenue (MR) Tolls. $\Delta \mathrm{p}_{\mathrm{a}}=\mathrm{g}_{\mathrm{a}}\left(\mathrm{x}_{\mathrm{a}}\right)^{\mathrm{n}}$

THEOREM 1C. ELASTIC DEMAND - NO BRAESS PARADOX IN TYPE H NETWORKS- The Braess Paradox does not occur for networks with single-power-law link costs that satisfy the Kirchhoff rules (Type H), even when demands are elastic.

As pointed out by a reviewer, it is worth noting that "With inelastic demand, total travel costs rise. With elastic demand, the supply (or cost) curve shifts to the left. Cost per trip rises, and consumers' surplus falls. But if demand is sufficiently elastic, usage may fall so much that total travel costs actually fall."

\section{ZERO MINIMAL REVENUE (MR) TOLLS FOR INELASTIC DEMAND AND POWER-LAW COSTS}

From Theorem 1A, we know that with inelastic demand, the same optimal flows occur both with and without $\mathrm{MC}$ tolls. Thus it is clear that zero toll yields the minimum revenue while maintaining optimal flows, so we have proved Theorem 2

THEOREM 2. INELASTIC DEMAND - ZERO MR TOLLS - For networks with single-power-law link costs that satisfy the Kirchhoff rules (Type H), for inelastic demands the Minimal Revenue (MR) tolls are stable at zero, independent of the level of demand.

\section{STABLE MINIMAL REVENUE (MR) TOLLS FOR INELASTIC DEMAND AND COSTS THAT INCLUDE FIXED COSTS PLUS POWER-LAW COSTS}

Here, we introduce the idea of a "stable" toll as one that does not change when conditions of price-inelastic demand change with time. Stable tolls are generally considered desirable because they are easier and cheaper to collect, and are predictable for the user. They allow the user to make easier route choices, and to make them in advance. Varying (i.e. un-stable) tolls, in response to small time dependent oscilla- 
The Braess Paradox in Minimal Critical Network: User Cost vs Network Flow

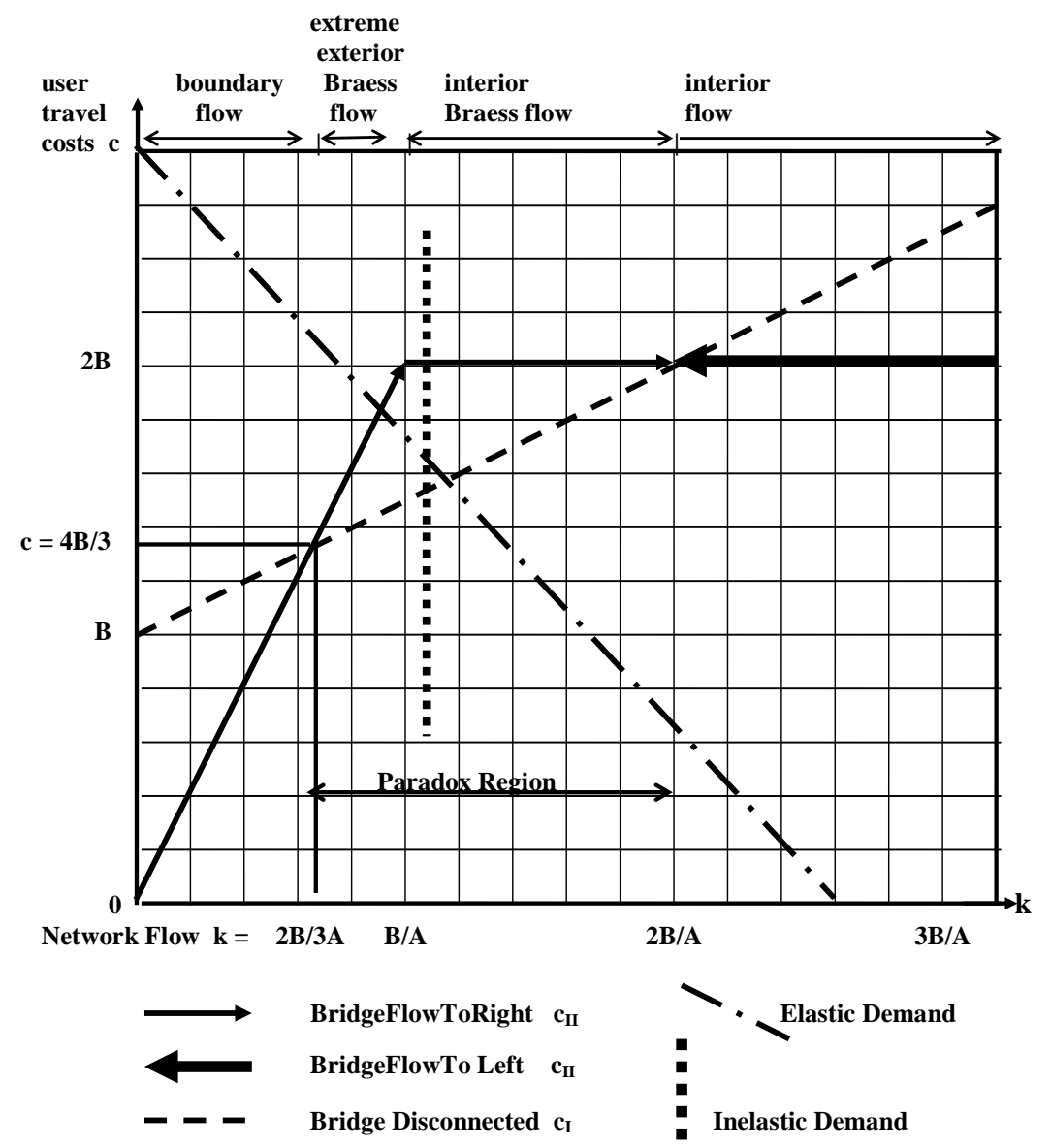

Graphical solution of the user equilibrium problem for the minimal Critical Network of Figure 1, showing total travel costs per user functions of the total network flow $\mathrm{k}$. The paradoxical region is a triangle within which the costs with bridge, c11, are higher than the costs without the bridge, c1. The labels at the top of the graph, for the various flow regions, are in the terminology of Frank (1981). The solid line shows the user travel cost c11, to travel from the Origin to the Destination when the bridge is used. All traffic uses the bridge to the right, with travel cost increasing linearly from 0 to $2 \mathrm{~B}$, as the total network flow increases from 0 to $\mathrm{B} / \mathrm{A}$. The bridge flow then decreases linearly to zero at a fixed travel cost $2 \mathrm{~B}$ as the total network flow increases to 2B/A. All additional traffic uses the bridge only to the left, if allowed, with fixed flow of B/A on each of the two non-bridged paths ( $\mathrm{L}$ and $\mathrm{R}$ in the notation of Frank (1981)) and fixed user cost 2B. Note that bridge flow to the left was not allowed in the original Brass network.

Fig. (2). This figure is adapted from figure 2 of Penchina, Claude M. (1997) "Braess Paradox: Maximal Penalty in a Minimal Critical Network", Transportation Research A31(5), pp. 379-388.

Here we have redrawn the original Fig. (2), and reproduced the original figure caption. Also, we added a downward sloping dash-dot line to indicate an example of elastic demand, and a vertical dotted line to indicate an example of inelastic demand.

tions in demand could also cause instabilities in the flow patterns.

Consider networks for which link costs include a fixed $\operatorname{cost} h_{j}$ in addition to the power-law congestion cost. Such networks are more reasonable representations of real traffic networks, than networks of type $\mathrm{H}$ with no fixed costs.

$c_{j}=h_{j}+g_{j}\left(x_{j}\right)^{n}$

The study of networks with non-zero fixed costs $h_{j}$ is more complicated. We find that it is useful to study first a very simple example network. Pedagogically, this study illustrates the stability of pricing for a case which is very easy to understand, and shows some of the ideas behind the MR pricing. More importantly, it is a simple counter-example to prove that the sufficient conditions for stability, which will be found, are not always necessary conditions.

\section{A. Example Network}

Consider the example of Fig. (1).

( $5 \mathrm{~A}$ a) It is seen by inspection that both paths must use link 1. Thus, for inelastic demand $d$, the flow through link 1 is

$\mathrm{x}_{1}=\mathrm{d}$

and can not be influenced by any toll, even if the cost function is much more complicated than that of Eq. (5.1). 
(5A b) The MR toll on this link is the minimum needed to assure SO flows. Since no toll can influence the flow, the minimum toll is clearly zero, independent of the (inelastic) network demand or the relationship between cost and flow in this link. Thus, we have proved Theorem 3

THEOREM 3. STABLE MINIMAL REVENUE (MR) TOLLS FOR INELASTIC DEMAND: NO NECESSARY CONDITIONS - For networks that satisfy the Kirchhoff rules, for inelastic demand, there is no cost-flow function that is always a necessary condition for stable MR tolls.

(5A c) Consider now links 2 and 3 of Fig. (1). It is clear that only the difference between the tolls on these links can influence the relative flows. Thus, for MR tolls, the smaller MC toll should be replaced by zero toll, and the larger one replaced by the difference between the two.

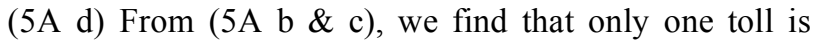
needed on only one link in the network of Fig. (1). This is an example of Dial's [5] result that MR always has at least one zero-toll path.

(5A e) For simplicity of the discussion, and without loss of generality, we let the MC toll (Tmc) be smaller on link 2 than on link 3 . The MR toll $\left(\mathrm{Tmr}_{3}\right)$ on link 3 is then

$\mathrm{Tmr}_{3}=\mathrm{Tmc}_{3}-\mathrm{Tmc}_{2}$

We need now examine the stability of $\mathrm{Tmr}_{3}$ under changes of inelastic demand $\mathrm{d}$.

(5A f) So long as the number of used links does not change, the variation of $\mathrm{Tmr}_{3}$ with demand $\mathrm{d}$ will be smooth, so we can use calculus to examine the changes. From Eqs (3.3) and (5.1) we get the same result Eq (3.4) as we did for networks of type $(\mathrm{H})$ in section 3 , since the derivative of the fixed cost $h$ is zero.

From Eq (5.3) the MR Toll on Link 3 is

$\mathrm{Tmr}_{3}=\mathrm{Tmc}_{3}-\mathrm{Tmc}_{2}=\mathrm{n}\left\{\mathrm{g}_{3}\left(\mathrm{x}_{3}\right)^{\mathrm{n}}-\mathrm{g}_{2}\left(\mathrm{x}_{2}\right)^{\mathrm{n}}\right\}$

From Kirchhoff's current law,

$\mathrm{x}_{3}+\mathrm{x}_{2}=\mathrm{d}$

( $5 \mathrm{~A} \mathrm{~g}$ ) One path unused. MR Tolls: If $\mathrm{x}_{2}=0$, the results are simple, and the MR tolls are all zero and thus all stable. $\mathrm{x}_{3}$ can not be zero unless $\mathrm{d}=0$, which is trivial and uninteresting.

(5A h) Both paths used. Equilibrium Costs. By Wardrop's principle (or Kirchhoff's voltage law) the user costs $\left(\mathrm{Cmc}_{\mathrm{j}}\right)$ including $\mathrm{MC}$ tolls

$\mathrm{Cmc}_{\mathrm{j}}=\mathrm{c}_{\mathrm{j}}+\mathrm{Tmc}_{\mathrm{j}}$

are equal for links 2 and 3 .

$\mathrm{Cmc}_{3}=\mathrm{Cmc}_{2}$

This must be true for any level of inelastic demand $\mathrm{d}$. Thus, if $\mathrm{d}$ changes

$\partial \mathrm{Cmc}_{3} / \partial \mathrm{d}=\partial \mathrm{Cmc}_{2} / \partial \mathrm{d}$

$\left\{\partial \mathrm{Cmc}_{3} / \partial \mathrm{x}_{3}\right\}\left\{\partial \mathrm{x}_{3} / \partial \mathrm{d}\right\}=\left\{\partial \mathrm{Cmc}_{2} / \partial \mathrm{x}_{2}\right\}\left\{\partial \mathrm{x}_{2} / \partial \mathrm{d}\right\}$

So

$\left\{\partial \mathrm{x}_{2} / \partial \mathrm{d}\right\} /\left\{\partial \mathrm{x}_{3} / \partial \mathrm{d}\right\}=\left\{\partial \mathrm{Cmc}_{3} / \partial \mathrm{x}_{3}\right\} /\left\{\partial \mathrm{Cmc}_{2} / \partial \mathrm{x}_{2}\right\}$

(5A i) Both Paths Used. Stability of MR tolls.

When inelastic demand $d$ changes $\partial \mathrm{Tmr}_{3} / \partial \mathrm{d}=\partial \mathrm{Tmc}_{3} / \partial \mathrm{d}-\partial \mathrm{Tmc}_{2} / \partial \mathrm{d}$

$=\left\{\partial \mathrm{Tmc}_{3} / \partial \mathrm{x}_{3}\right\}\left\{\partial \mathrm{x}_{3} / \partial \mathrm{d}\right\}-\left\{\partial \mathrm{Tmc}_{2} / \partial \mathrm{x}_{2}\right\}\left\{\partial \mathrm{x}_{2} / \partial \mathrm{d}\right\}$

From Eq (5.10) we find

$\partial \mathrm{Tmr}_{3} / \partial \mathrm{d}=\left\{\partial \mathrm{Cmc}_{3} / \partial \mathrm{x}_{3}\right\}\left\{\partial \mathrm{x}_{3} / \partial \mathrm{d}\right\} \mathrm{X}$

$\left[\left(\left\{\partial \mathrm{Tmc}_{3} / \partial \mathrm{x}_{3}\right\} /\left\{\partial \mathrm{Cmc}_{3} / \partial \mathrm{x}_{3}\right\}\right)-\left(\left\{\partial \mathrm{Tmc}_{2} / \partial \mathrm{x}_{2}\right\} /\left\{\partial \mathrm{Cmc}_{2} / \partial \mathrm{x}_{2}\right\}\right)\right](5.12)$

From Eqs (3.1), (3.3), (3.4), (5.6) we find that the term in square brackets [] is zero, so

$\partial \mathrm{Tmr}_{3} / \partial \mathrm{d}=0$

so the MR toll is totally stable for all flows where both paths are used.

(5A j) Stability of MR tolls for example network - Summary.

A toll is needed only on link 3 . This $\mathrm{Tmr}_{3}$ is piecewise stable. $\mathrm{Tmr}_{3}=0$ for all demands small enough for only one path to be used, and then constant again at $\mathrm{Tmr}_{3}=\mathrm{Tmc}_{3}-$ $\mathrm{Tmc}_{2}$ when both paths are used. This proof easily extends to any integer number $m$ (not just 2) of links in parallel. This result also leads us to conjecture a new theorem 4 , which is then proved in section $5 \mathrm{~B}$.

THEOREM 4. PIECEWISE STABLE MINIMAL REVENUE (MR) TOLLS FOR INELASTIC DEMAND: SUFFICIENT CONDITIONS - For networks that satisfy the Kirchhoff rules, a cost function

$c_{j}=h_{j}+g_{j}\left(x_{j}\right)^{n}$ on each link $j$, is sufficient (but not always necessary) for piecewise stable MR tolls. The tolls may change from one stable value to another as the number of used links in the network changes when the demand changes.

\section{B. General Proof of Theorem 4}

(5B a) We start this proof with a situation for which we have equilibrium with MR tolls for a network as in theorem 4 , that satisfies the Kirchhoff rules, and has cost functions $c_{j}$ on links $\mathrm{j}$, as in Eq (5.1) repeated here

$\mathrm{c}_{\mathrm{j}}=\mathrm{h}_{\mathrm{j}}+\mathrm{g}_{\mathrm{j}}\left(\mathrm{x}_{\mathrm{j}}\right)^{\mathrm{n}}$

If demands change, the link flows $\mathrm{x}_{\mathrm{j}}$ change, and the corresponding $\mathrm{MC}$ tolls also change, as in $\mathrm{Eq}$ (3.3) repeated here

$T \mathrm{mc}_{\mathrm{j}}=\mathrm{x}_{\mathrm{j}} \partial \mathrm{c}_{\mathrm{j}} / \partial \mathrm{x}_{\mathrm{j}}$

For a nearby range of demands over which the integer number $m$ of used links does not change, we expect smooth changes so we can use calculus for our analysis of corresponding changes in MR tolls Tmr, which turn out to be zero for fixed $\mathrm{m}$.

(5B b) Differentiating Eq (5.6) for the link costs $\mathrm{Cmc}_{\mathrm{j}}$ with MC tolls, we find

$\partial \mathrm{Cmc}_{\mathrm{j}} / \partial \mathrm{x}_{\mathrm{j}}=\partial \mathrm{c}_{\mathrm{j}} / \partial \mathrm{x}_{\mathrm{j}}+\partial \mathrm{Tmc}_{\mathrm{j}} / \partial \mathrm{x}_{\mathrm{j}}$

and using Eqs (3.1) and (3.3), we find

$\partial c_{j} / \partial x_{j}=n g_{j}\left(x_{j}\right)^{n-1}$

$\partial \mathrm{Tmc}_{\mathrm{j}} / \partial \mathrm{x}_{\mathrm{j}}=\mathrm{n}_{2} \mathrm{~g}_{\mathrm{j}}\left(\mathrm{x}_{\mathrm{j}}\right)^{\mathrm{n}-1}$

so

$\partial \mathrm{Cmc}_{\mathrm{j}} / \partial \mathrm{x}_{\mathrm{j}}=\mathrm{n}(\mathrm{n}+1) \mathrm{g}_{\mathrm{j}}\left(\mathrm{x}_{\mathrm{j}}\right)^{\mathrm{n}-1}$ 
(5B c) The derivatives of $\mathrm{c}_{\mathrm{j}}, \mathrm{Tmc}_{\mathrm{j}}$, and $\mathrm{Cmc}_{\mathrm{j}}$, are independent of $h_{j}$, so that changes in costs are identical to those which would be obtained with $h_{j}=0$, for which the flows were shown to be system optimal in section 3 , Theorem 1A. The increases in link flows due to increases in demand are thus also independent of $h_{j}$ and are the same system optimal extra flows with or without tolls, just as the total flows were system optimal in section 3. Thus, Theorem 1A applies to the extra demand here, just as it applies to the total demand in section 3 . Just as in Theorem 1C of section 3 where the total MR tolls were all zero, here the extra MR tolls are zero. Thus, the MR tolls are stable until the demands increase enough to change the number of used links, causing a discontinuous change in flows and invalidating our calculus analysis. For example, if there is zero flow in one or more links at low demand, the flow in a link may change from zero to non-zero as the demand grows. The flow-vs-demand curve for that link would have a discontinuous slope, and invalidate the analysis based on derivatives (differential calculus), thus leading to a discontinuous change in the MR tolls. From there on, the MR tolls are again stable at a new level as long as m remains constant, so the $\mathrm{Tmr}_{\mathrm{j}}$ are Piecewise Constant. This completes the proof of Theorem 4.

\section{SUMMARY AND DISCUSSION}

Based on the system optimality of flows under Marginal Cost MC tolls, we have shown a simple proof of the nonoccurrence of the Braess Paradox in networks with singlepower-law congestion costs and no fixed costs. We extended the theorem to show that it applies to elastic demands as well as inelastic demands.

We then used the ideas of optimality, from the study of the Braess Paradox, to find new theorems regarding Minimal Revenue MR tolls in networks with inelastic demands. The theorems $(2,3 \& 4)$ on MR tolls with inelastic demand can not be extended to elastic demand [11]; the basic idea of MR pricing does not truly apply when demand is elastic. We found [11] that link costs which include a fixed cost and singlepower-law congestion costs (such as the popular BPR function [12]) are sufficient (but not always necessary) to cause MR tolls to be piecewise stable, changing discontinuously only when the number of used links changes. This piecewise stability can reduce collection costs, and makes planning simpler for the system managers and users. If the fixed costs are zero on all links, then the MR tolls are zero (totally stable) for all levels of inelastic demand. Although zero fixed costs are not very realistic for most traffic networks, it could be a reasonable approximation to networks with very heavy congestion on short links.

\section{ABBREVIATIONS}

$\mathrm{C}-\mathrm{K}=$ Calvert $\&$ Keady (Reference [1])
$\mathrm{GC}=$ Generalized Cost $=$ time plus the time-equivalent of other costs. (Reference [5])

$\mathrm{MC}=$ Marginal Cost

$\mathrm{MR}=$ Minimal revenue (References $[5,11])$

$\mathrm{SO}=$ System Optimal

$\mathrm{UO}=$ User Optimal

\section{ACKNOWLEDGEMENTS}

We wish to thank Bruce Calvert and Grant Keady for several very useful discussions about the Braess Paradox and for their help in understanding the proofs of their theorems. We thank Benjamin Heydecker for first bringing the literature on MR pricing to our attention. We thank Robin Lindsey for many useful discussions, and many detailed editorial comments and references to the literature. We thank also an anonymous reviewer of our previous research paper [11] on Minimal Revenue MR tolls, for some very useful editorial comments and for suggesting that I publish the proofs of theorems which were referred to there without proof.

The research received some support from Gilora Associates.

\section{REFERENCES}

[1] B. Calvert and G. Keady, "Braess's paradox and power-law nonlinearities in networks" J. Austral. Math. Soc. Ser. B 35, pp. 1-22, 1993.

[2] D. Braess, "Ueber ein Paradoxen der Verkehrsplannung", Unternehmensforschung, vol. 12, pp. 258-268, 1968.

[3] G.R. Kirchhoff, Vorlesungen über mathematische Physik (1876-94) see http://www-gap.dcs.st-and.ac.uk/ history/Mathematicians/Kirchhoff.html, http://www.twysted-pair.com/ohms.htm and http://www.phys.ualberta.ca/ gingrich/phys395/notes/node10.html (See also any standard textbooks onn electric circuits).

[4] J. G. Wardrop, "Some theoretical aspects of road traffic research", Proc. Inst. Civil Eng., vol. 1, pp. 325-378, 1952.

[5] R.B. Dial, "Minimal-revenue congestions pricing part I: A fast algorithm for the single-origin case", Transport. Res. B 33, pp. 189-202, 1999.

[6] R. Barro, and P. M. Romer, "Ski-lift pricing, with applications to labor and other markets", Am. Econ. Rev., vol. 77(5), pp. 875-890, 1987.

[7] T. Cowen, and A. Glazer, "Ski-lift pricing with applications to labor and other markets: Comment", Am. Econ. Rev., vol. 81(1), pp 376$377,1991$.

[8] Nathan H. Gartner, "Optimal Traffic Assignment with Elastic Demands: A Review. Part I Analysis Framework", Transport. Sci., vol. 14, pp. 174-191, 1980.

[9] I. Milchtaich, "Network Topology and the Efficiency of Equilibrium", Games Econ. Behav., vol. 57, pp. 321-346, 2006.

[10] C. M. Penchina, "Braess Paradox: Maximal Penalty in a Minimal Critical Network", Transport. Res., vol. A31(5), pp. 379-388, 1997.

[11] C. M. Penchina, "Minimal-revenue congestion pricing: some more good-news and bad-news", Transport. Res. B: Method., vol. 38, pp. 559-570, 2004.

[12] BPR, BUREAU OF PUBLIC ROADS Traffic assignment manual. : U.S. Department of Commerce, Washington, DC June 1964. 